\title{
ASSESSMENT OF BODY COMPOSITION AMONG CURRENT CZECH PROFESSIONAL SOCCER PLAYERS WITH DIFFERENT PERFORMANCE LEVELS
}

original paper

( ) University School of Physical Education in Wroclaw

DOI: https://doi.org/10.5114/hm.2018.81016

\section{LUKAS ONDRA, MICHAL KUDLACEK, MARTIN SIGMUND}

Palacký University Olomouc, Olomouc, Czech Republic

\begin{abstract}
Purpose. To assess differences in body composition among professional soccer players with different performance levels and determine whether there is an association between body fat mass (BFM) and soccer-specific performance in professional soccer players.

Methods. Anthropometric and biometric data were evaluated for 203 participants (soccer players, $n=135$; comparative group, $n=68$ ). Soccer players were divided in accordance with the final standings of the Czech First League in the $2016-2017$ season (top 3 teams: promoted group, $n=59$; last 3 teams: relegated group, $n=76$ ).

Results. No significant differences were found in fat free mass (FFM) $(p=0.714)$, skeletal muscle mass $(\mathrm{SMM})(p=0.67)$, height $(p=0.413)$, or weight $(p=0.132)$ between soccer players with different performance levels. Players with a high performance level reported significantly higher body fat percentage $(p<0.001, d=0.880)$ and $\mathrm{BFM}(p<0.001, d=0.908)$ than those with a low performance level.

Conclusions. There were no significant differences in body height, body weight, FFM, or SMM between the soccer players with different performance levels. However, significant differences were found in BFM: high performance players presented higher BFM than low performance players. Thus, BFM is not a limiting factor in soccer-specific performance of professional soccer players. Soccer coaches should pay more attention to the tactical and technical part of soccer-specific performance. Key words: eperformance analysis, soccer physiology, anthropometry
\end{abstract}

\section{Introduction}

Soccer success is most likely indicated by the collective performance of its players throughout the season [1]. To enhance the performance of soccer players, coaches must focus on various factors, such as technical/biomechanical, tactical, mental, and physiological aspects [2]. In soccer, there are specific fitness requirements for every player according to their playing position on the pitch [3, 4]. The production of superior performance during a sport-specific task, such as soccer playing, is determined, beside other factors, by muscular strength [5]. Muscular strength is strongly correlated to soccerspecific movements and motion patterns like sprinting, agility performance, and jumping [5].

Although the importance of body composition for sports performance has long been known [6], currently, soccer has become faster, with higher intensity and playing performance requirements than over the past decades [2]. This brings even higher demands on the physiological factors of soccer players, such as body composition [7], on one hand, and on tactical factors, such as direct style of play with a lot of shoot attempts [8], on the other.

Tactical factors, a component of technical effectiveness, indicate the success of a team in a competition. The activity patterns (i.e. high intensity running distance, sprint distance, etc.), which are influenced by the physical fitness factors described in previous studies $[9,10]$, may reduce physical performance in later stages of a game [11]. These conditions lead to loss of overall performance and to fatigue [11]. Thus, it is important to select and then implement suitable and effective tactical strategies [12] for future success in a competition.

Correspondence address: Lukáš Ondra, Department of Natural Sciences in Kinanthropology, Faculty of Physical Culture, Palacký University Olomouc, Tř. Míru 117, 77147 Olomouc, Czech Republic, e-mail: lukas.ondra@upol.cz

Received: October 6, 2018

Accepted for publication: December 12, 2018

Citation: Ondra L, Kudlacek M, Sigmund M. Assessment of body composition among current Czech professional soccer players with different performance levels. Hum Mov. 2018;19(5)special/issue:60-64; doi: https://doi.org/10.5114/hm.2018.81016. 
Professional soccer players cover a distance of ca. $10-12 \mathrm{~km}$ during a competition match $[2,13]$. The most frequent activities performed during a competition match are walking and jogging in low intensity [13], and each professional soccer player performs 10001400 principally short activities that change every 4-6 seconds [14]. On an average, soccer matches involve a short sprint every 90 seconds, lasting for 2-4 seconds [15]. Sprinting represents up to $11 \%$ of the total distance covered during a competition match [14]. Small changes in upper body fat mass (BFM) may have a major impact on the ability to perform soccer-specific anaerobic movements, such as vertical jump, short distance sprint, or quick change of direction [16].

The current study aimed to assess (1) differences in body composition among professional soccer players with different performance levels and (2) the association between BFM and soccer-specific performance in professional soccer players.

\section{Material and methods}

\section{Participants}

In total, we analysed data collected from 203 participants (soccer players, $n=135$; comparative group, $n=68$ ). Soccer players were divided into 2 groups according to the performance level of their teams (promoted or relegated) on the basis of the final standings of the Czech First League in the 2016-2017 season. The top 3 teams (promoted) consisted of 59 soccer players and the bottom 3 teams (relegated) involved 76 players. The comparative group comprised university students. The anthropometric and biometric information for each group has been presented in Table 1 . Considering the performance level and the performance differences, the top 3 teams of the Czech First League gained promotion to the UEFA competitions and the last 2 teams were relegated to the Czech National Soccer League ( $2^{\text {nd }}$ league).

All the professional players participated in the regular training sessions and scheduled matches of the competition during the 2016-2017 season. The subjects in the comparative group were involved in recreational sport activities only or did not engage in any sport. Additionally, they had not engaged in any competitive sport training in the past.

\section{Procedure}

The bioelectrical impedance analysis was carried out in accordance with the recommended domestic and international standards of the International Society for the Advancement of Kinanthropometry [17, 18]. Football players were tested in one week after the end of the season (i.e. after the last competition match). Each of the participating teams was examined during one day. Body height was measured with a stadiometer (Leicester High Measure MK II, Leicester, Great Britain). Total body weight, fat free mass (FFM), skeletal muscle mass (SMM), BFM, and body fat percentage $(\mathrm{BF} \%)$ were determined with the use of a calibrated InBody 230 bioelectrical impedance device (Biospace, Seoul, Korea) in the morning, after overnight fasting, after emptying the bladder and in euhydrated state. The participants were instructed not to perform any physical activity for 24 hours before the body composition measurement. All the participants were examined while wearing light underwear.

\section{Statistical analyses}

Data obtained in the investigation were analysed with the Statistica software (version 10; Statsoft, Tulsa, OK, USA). The data were not normally distributed, as verified with the Kolmogorov-Smirnov test. Krus-

Table 1. Descriptive anthropometric and biometric data of the participants

\begin{tabular}{|c|c|c|c|}
\hline & $\begin{array}{c}\text { Promoted group }(n=59) \\
\text { mean }(S D)\end{array}$ & $\begin{array}{c}\text { Relegated group }(n=76) \\
\text { mean }(S D)\end{array}$ & $\begin{array}{c}\text { Comparative group }(n=68) \\
\text { mean }(S D)\end{array}$ \\
\hline Age (years) & $25.59(4.34)$ & $22.56(4.51)$ & $26.15(8.07)$ \\
\hline Height (cm) & 183.12 (5.93) & 183.88 (5.77) & 178.68 (6.49) \\
\hline Weight (kg) & 80.78 (7.17) & 79.07 (7.68) & $77.10(8.90)$ \\
\hline FFM (kg) & $70.25(6.72)$ & $71.11(7.05)$ & $65.15(8.02)$ \\
\hline SMM (kg) & $40.29(4.01)$ & $40.82(4.13)$ & $37.03(4.82)$ \\
\hline BFM (kg) & $10.53(2.94)$ & $7.96(3.04)$ & $19.85(10.23)$ \\
\hline $\mathrm{BF} \%$ & $13.03(3.41)$ & $10.01(3.44)$ & $22.36(7.20)$ \\
\hline
\end{tabular}

FFM - fat free mass, SMM - skeletal muscle mass, BFM - body fat mass, BF\% - body fat percentage 
L. Ondra, M. Kudlacek, M. Sigmund, Body composition among professional soccer player

kal-Wallis ANOVA was used to investigate differences between all the groups. The differences in the anthropometric variables between participants with different performance levels were determined with the nonparametric Mann-Whitney $U$ test. The significance level was set at $\alpha=0.05$. The effect size was assessed by Cohen's $d$ as follows: small effect: $0.20 \leq d<0.50$, medium effect: $0.50 \leq d<0.80$, and large effect: $d \geq$ $0.80[19]$.

\section{Ethical approval}

The research related to human use has been complied with all the relevant national regulations and institutional policies, has followed the tenets of the Declaration of Helsinki, and has been approved by the authors' institutional review board or an equivalent committee.

\section{Informed consent}

Informed consent has been obtained from all individuals included in this study.

\section{Results}

The results showed (Table 2) that the promoted group reported significantly higher $\mathrm{BF} \%(p<0.001$, $d=0.880)$ and BFM $(p<0.001, d=0.908)$ than the relegated group. However, we found no significant differences between the two groups in terms of FFM ( $p=$ $0.714)$, SMM ( $p=0.67)$, height $(p=0.413)$, or weight $(p=0.132)$.

As expected, both groups of professional soccer players had significantly lower $\mathrm{BF} \%\left(p_{\text {promoted }}<0.001\right.$, $p_{\text {relegated }}$ $<0.001)$ and BFM $\left(p_{\text {promoted }}<0.001, p_{\text {relegated }}<0.001\right)$, and significantly higher FFM $\left(p_{\text {promoted }}<0.001, p_{\text {relegated }}\right.$ $<0.001)$ and SMM $\left(p_{\text {promoted }}<0.001, p_{\text {relegated }}<0.001\right)$ as compared with the comparative group (Table 2).

\section{Discussion}

We evaluated the body composition of professional soccer players with different performance levels; however, we did not consider the playing position of the players. Instead, we analysed the data with respect to the performance level of the players according to the final standings of their teams in the league. Moreover, we assessed whether BFM was a limiting factor for soccer-specific performance in professional soccer players.

The present findings revealed no differences in FFM or SMM between the promoted and relegated groups. As muscular strength is strongly correlated to soccerspecific movements and motion patterns [5], the lack of differences in FFM or SMM indicates that these factors are related to the performance level of professional soccer players. Though the relegated group comprised younger players than the promoted group, there were no significant differences in weight and height. This finding is similar to that of a study evaluating the body composition of members of U21 and U18 squads of an English Premier League soccer team [20]. In the present study, the only significant differences were found in BFM and BF\%, with the promoted group showing higher values than the relegated group.

So far, research on the relationship between body fat and performance has been scarce. Body composition parameters such as BFM may contribute to agility performance. Agility, which can be defined as rapid whole-body movement with change of velocity or direction [21], is considered as a key part of performance in team sports [22, 23], such as soccer. It is assumed that greater force production is required to produce a given change in velocity or direction in athletes with higher $\mathrm{BF} \%$. A study evaluating soccer players' agility [24] revealed that players who performed better in the change of direction speed (CODS) test tended to

Table 2. Differences in selected anthropometric parameters between groups

\begin{tabular}{ccc}
\hline $\begin{array}{c}p \text { value } \\
\text { (promoted } \times \text { relegated) }\end{array}$ & $\begin{array}{c}p \text { value } \\
\text { (promoted } \times \text { comparative) }\end{array}$ & $\begin{array}{c}p \text { value } \\
\text { (relegated } \times \text { comparative) }\end{array}$ \\
\hline 0.413 & 0.001 & 0.001 \\
0.133 & 0.002 & 0.076 \\
0.714 & 0.001 & 0.001 \\
0.670 & 0.001 & 0.001 \\
0.001 & 0.001 & 0.001 \\
0.001 & 0.001 & 0.001
\end{tabular}

FFM - fat free mass, SMM - skeletal muscle mass, BFM - body fat mass, BF\% - body fat percentage 0.001 indicates $p<0.001$ 
have lower BFM. However, there was no direct correlation between CODS and $\mathrm{BF} \%$. Though the importance of lower $\mathrm{BF} \%$ in maximizing CODS is hypothesised, the relationship between these variables remains unclear. Nevertheless, muscle mass and other leg strength qualities have been highlighted as some of the important factors influencing agility [25].

It is well known that every professional soccer player is required to meet some physiological and morphological demands [2, 26]. Despite the fact that body composition influences performance in soccer [7], according to recent studies $[8,12,27]$, technical and tactical aspects seem to be more important. A study that evaluated the influence of high intensity running on overall team success [11] reported that high levels of physical performance was not as important to success in competition as the overall intensity of work in relation to the tactical and technical effectiveness of the team. Therefore, a direct style of play with a lot of shooting attempts [8] should be adopted by teams to ensure success.

On the basis of the statistics of the 2016-2017 season of the Czech First League [28], the top 3 teams (promoted group) had a higher average number of shots on target (153 shots) than the bottom 3 teams (relegated group, 112 shots). Similarly, the average number of shots off target was higher for the promoted group (418 shots) than that for the bottom 3 teams (324 shots). These results confirm the findings that successful teams need to have a higher number of total shots and shots on target [29,30]. One study [12] identified performance indicators that discriminated winning teams and drawing and losing teams in the UEFA Champions League. Another study [27] determined specific performance indicators that distinguished top clubs on the basis of significantly different pitch action performance in the Spanish Soccer League. Both studies showed that top teams had significantly higher values of total shots and shots on target than the teams with lower performance levels.

The present results showed that higher BFM was not a limiting factor for soccer-specific performance in professional soccer players. The tactical and technical aspects of soccer-specific performance seems to be important to success in professional soccer competitions. To fulfil these tactical and technical demands, every professional soccer player has to meet some specific physiological requirements. Experience and maturity, represented by the number of years of playing at the professional level, may also be factors influencing soccer-specific performance because experienced soccer players are likely to adapt more quickly to changing conditions during a competitive match.

\section{Conclusions}

In summary, the present results revealed no significant differences in body height, body weight, FFM, or SMM between the top and bottom 3 soccer teams (promoted and relegated groups, respectively) of the Czech First League. However, there were significant differences in BFM: players with better performance (promoted group) had higher BFM than those with low performance (relegated group). Therefore, we conclude that higher BFM is not a limiting factor for soccer-specific performance in professional soccer players.

\section{Acknowledgements}

The authors would like to thank the managers who granted permission for us to assess their first team squads.

This work was supported by a grant by Palacký University Olomouc (No. IGA_FTK_2018_008).

\section{Disclosure statement}

No author has any financial interest or received any financial benefit from this research.

\section{Conflict of interest}

The authors state no conflict of interest.

\section{References}

1. Oberstone J. Differentiating the top English Premier League football clubs from the rest of the pack: identifying the keys to success. J Quant Anal Sports. 2009; 5(3):10; doi: 10.2202/1559-0410.1183.

2. Stølen T, Chamari K, Castagna C, Wisløff U. Physiology of soccer: an update. Sports Med. 2005;35(6):501536; doi: 10.2165/00007256-200535060-00004.

3. Semjon M, Botek M, Svozil Z, McKune AJ. Positional differences in the cardiorespiratory, autonomic, and somatic profiles of professional soccer players. Acta Gymn. 2016;46(2):90-96; doi: 10.5507/ag.2016.008.

4. Hencken C, White C. Anthropometric assessment of Premiership soccer players in relation to playing position. Eur J Sport Sci. 2006;6(4):205-211; doi: 10.1080/ 17461390601012553.

5. Suchomel TJ, Nimphius S, Stone MH. The importance of muscular strength in athletic performance. Sports Med. 2016;46(10):1419-1449; doi: 10.1007/s40279016-0486-0.

6. Leedy HE, Ismail AH, Kessler WV, Christian JE. Relationships between physical performance items and body composition. Res Q. 1965;36(2):158-163; doi: 10.1080/ 10671188.1965.10614674.

7. Sutton L, Scott M, Wallace J, Reilly T. Body composition of English Premier League soccer players: influence of playing position, international status, and ethnicity. 
L. Ondra, M. Kudlacek, M. Sigmund, Body composition among professional soccer player

J Sports Sci. 2009;27(10):1019-1026; doi: 10.1080/ 02640410903030305.

8. Kite CS, Nevill A. The predictors and determinants of inter-seasonal success in a professional soccer team. J Hum Kinet. 2017;58(1):157-167; doi: 10.1515/hukin2017-0084.

9. Mujika I, Santisteban J, Impellizzeri FM, Castagna C. Fitness determinants of success in men's and women's football. J Sports Sci. 2009;27(2):107-114; doi: 10.1080/02640410802428071.

10. Arnason A, Sigurdsson SB, Gudmundsson A, Holme I, Engebretsen L, Bahr R. Physical fitness, injuries, and team performance in soccer. Med Sci Sports Exerc. 2004;36(2):278-285;doi:10.1249/01.MSS.0000113478. 92945.CA.

11. Di Salvo V, Gregson W, Atkinson G, Tordoff P, Drust B. Analysis of high intensity activity in Premier League soccer. Int J Sports Med. 2009;30(3):205-212; doi: 10.1055/s-0028-1105950.

12. Lago-Peñas C, Lago-Ballesteros J, Rey E. Differences in performance indicators between winning and losing teams in the UEFA Champions League. J Hum Kinet. 2011;27(1);135-146; doi: 10.2478/v10078-011-0011-3.

13. Rampinini E, Coutts AJ, Castagna C, Sassi R, Impellizzeri FM. Variation in top level soccer match performance. Int J Sports Med. 2007;28(12):1018-1024; doi: 10.1055/s-2007-965158.

14. Mohr M, Krustrup P, Bangsbo J. Match performance of high-standard soccer players with special reference to development offatigue. J Sports Sci. 2003;21(7):519528; doi: 10.1080/0264041031000071182.

15. Bangsbo J, Nørregaard L, Thorsø F. Activity profile of competition soccer. Can J Sport Sci. 1991;16(2):110-116.

16. Inacio M, Dipietro L, Visek AJ, Miller TA. Influence of upper-body external loading on anaerobic exercise performance. J Strength Cond Res. 2011;25(4):896902; doi: 10.1519/JSC.0b013e318208afb9.

17. Heyward VH, Wagner DR. Applied body composition assessment, $2^{\text {nd }}$ ed. Champaign: Human Kinetics; 2004.

18. Ward LC. Segmental bioelectrical impedance analysis: an update. Curr Opin Clin Nutr Metab Care. 2012; 15(5):424-429; doi: 10.1097/MCO.0b013e328356b944.

19. Cohen J. Statistical power analysis for the behavioral sciences. New York: Lawrence Erlbaum Associates; 1988.

20. Milsom J, Naughton R, O’Boyle A, Iqbal Z, Morgans R, Drust B, et al. Body composition assessment of English Premier League soccer players: a comparative DXA analysis of first team, U21 and U18 squads. J Sports Sci. 2015;33(17):1799-1806; doi: 10.1080/02640414. 2015.1012101.

21. Sheppard JM, Young WB. Agility literature review: classifications, training and testing. J Sports Sci. 2006; 24(9):919-932; doi: 10.1080/02640410500457109.

22. Paul DJ, Gabbett TJ, Nassis GP. Agility in team sports: testing, training and factors affecting performance. Sports Med. 2016;46(3):421-442; doi: 10.1007/s40279015-0428-2.
23. Rauter S, Coh M, Vodicar J, Zvan M, Krizaj J, Simenko J, et al. Analysis of reactive agility and change-ofdirection speed between soccer players and physical education students. Hum Mov. 2018;19(2):68-74; doi: 10.5114/hm.2018.74061.

24. Reilly T, Williams AM, Nevill A, Franks A. A multidisciplinary approach to talent identification in soccer. J Sports Sci. 2000;18(9):695-702; doi: 10.1080/ 02640410050120078.

25. Young WB, James R, Montgomery I. Is muscle power related to running speed with changes of direction? J Sports Med Phys Fitness. 2002;42(3):282-288.

26. Burdukiewicz A, Pietraszewska J, Stachoń A, Chromik K, Goliński D. The anthropometric characteristics of futsal players compared with professional soccer players. Hum Mov. 2014;15(2):93-99; doi: 10.2478/ humo-2014-0008.

27. Lago-Ballesteros J, Lago-Peñas C. Performance in team sports: identifying the keys to success in soccer. $\mathrm{J}$ Hum Kinet. 2010;25(1):85-91; doi: 10.2478/v10078010-0035-0.

28. Football League Association, ePojisteni.cz league 2016/ 2017: detailed statistics, InStat, 2017 [in Czech]. Available from: https://www.fortunaliga.cz/statistiky?unit $=2 \&$ status $=0 \&$ season $=2017 \&$ club $=0 \&$ game_limit $=0$ \&nationality $=\&$ age $=0 \&$ order $=0$ \&order_dir $=0 \&$ list_ number $=0$ \& parameter $=1$.

29. Castellano J, Casamichana D, Lago C. The use of match statistics that discriminate between successful and unsuccessful soccer teams. J Hum Kinet. 2012;31(1): 139-147; doi: 10.2478/v10078-012-0015-7.

30. Armatas V, Yiannakos A, Zangelidis G, Skoufas D, Papadopoulou S, Fragkos N. Differences in offensive actions between top and last teams in Greek first soccer division. A retrospective study 1998-2008. J Phys Educ Sport. 2009;23(2):1-5. 